\title{
Order and defectivity nanometrology by image processing and analysis of sub-20 nm BCPs features for lithographic applications
}

\author{
C. Simão, ${ }^{* \mathrm{a}}$ D. Tuchapsky, ${ }^{\mathrm{b}}$ W. Khunsin, ${ }^{\mathrm{a}, \mathrm{c}}$ A. Amann, ${ }^{\mathrm{b}}$ M. A. Morris ${ }^{\mathrm{d}, \mathrm{e}}$ and C. M. Sotomayor Torres ${ }^{\mathrm{a}, \mathrm{f}}$ \\ ${ }^{a}$ Catalan Institute of Nanoscience and Nanotechnology ICN2, Campus de la UAB, Barcelona 08193, \\ Spain; ${ }^{b}$ School of Mathematics and the Tyndall National Institute, UCC, Cork Ireland; ${ }^{c}$ Green \\ Nanotechnology Research Centre, Research Institute for Electronic Science, Hokkaido University, 001- \\ 0021 Sapporo, Japan; ${ }^{\mathrm{d}}$ School of Chemistry and the Tyndall National Institute, ${ }^{\mathrm{e}}$ UCC, Cork Ireland; \\ Centre for Research on Adaptive Nanostructures and Nanodevices, TCD, Ireland; ${ }^{\mathrm{f}}$ Catalan Institute of \\ Research and Advanced Studies, Barcelona 08010 , Spain;
}

\begin{abstract}
The line patterns obtained by the self-assembly of the block copolymer (BCP) polystyrene-b-polyethylene oxide (PS-b-PEO) was investigated. The hexagonal PS-b-PEO 42k-11.5k in a thin film was solvent annealed in a chlorophorm saturated atmosphere for three different annealing times. The microphase segregation of this BCP returned 18nm cylinders of PEO through the PS matrix, with an approximately $40 \mathrm{n}$ periodicity, as expected. Under chlorophorm vapours, the PEO cylinders oriented perpendicular to the silicon substrate while increasing the annealing time. These cylinders formed linear patterns with different alignment. To achieve insights about the percentage of alignment, defect type pareto and density, and order quantification to compare the three annealing recipes, the samples were analysed with innovative image analysis software specifically developed in our laboratory to identify elements and defects of line arrays from block copolymer self-assembly. From this technique, it was extracted dimensional metrology estimating pitch size and placement error, and the linewidth of the lines was estimated. Secondly, the methodology allows identification and quantification of typical defects observable in BCP systems, such as turning points, disclination or branching points, break or lone points and end points. The defect density and the quantification of the alignment were estimated using our technique. The methodology presented here represents a step forward in dimensional metrology and defect analysis of BCP DSA systems and can be readily used to analyze other lithographic or non-lithographic patterns.
\end{abstract}

\section{Introduction}

Extending the limits of nanofabrication to the nanometre scale had impact on specialized metrology suitable both to the dimensions and novel materials requirements. ${ }^{1-3}$ At the sub-20 nm scale nanostructures, fabrication costs have triggered the search of alternative fabrication techniques. One of the most promising technologies is directed self-assembly of block copolymers, ${ }^{4-5}$ which are valuable systems to create hexagonal and linear patterns on surface, only by tuning substrate surfaces properties. ${ }^{6-8}$

This self-assembly type technology can result in characteristic fingerprint patterns to highly controllably aligned lines patterns on surface using state-of-the-art directed self-assembly (DSA) techniques. ${ }^{9-12}$ These procedures rely on the control of surface properties, either chemical or topological, to create a confinement commensurable with the polymer chains and have a direct impact in their regular arrangement.

Dimensional Optical Metrology and Inspection for Practical Applications III, edited by

Kevin G. Harding, Toru Yoshizawa, Song Zhang, Proc. of SPIE Vol. 9110, 91100R

(C) 2014 SPIE · CCC code: 0277-786X/14/\$18 - doi: 10.1117/12.2050182 
One of the biggest challenges towards industrial adaptation that remains to be addressed is the estimation of defect density on these systems. Here we present an innovative methodology to analyse linear patterns generated by BCP DSA, ${ }^{13}$ based on an image analysis software that identifies the line elements and is able to provide valuable identification of typical defects from BCP DSA, namely turns, dislocations, lone and branching points, all of which are crucial for the optimization and tailoring of the assembly process to obtained desired structures.. The defect density and the quantification of the alignment are then estimated based on the statistical data gathered by the image analysis.

\section{Experimental}

\subsection{Materials}

Block copolymer polystyrene-b-polyethylene oxide (PS-b-PEO) $\left(42 \mathrm{~kg} \cdot \mathrm{mol}^{-1}-11.5 \mathrm{~kg} \cdot \mathrm{mol}^{-1}\right)$ and solvents toluene and chloroform were used as purchased from Polymer Source Inc. (USA). $100 \mathrm{~mm}$ silicon wafers (p type, boron, $<100>, 10-20$ $\mathrm{ohm} / \mathrm{cm})$ (Silicon Materials Inc. USA), were used as purchased.

\subsection{Block copolymer sample preparation}

A solution of PS-b-PEO 1\% (w/w) in toluene was prepared and filtered through a $200 \mathrm{~nm}$ pore size syringe filter. This solution was dispensed over silicon substrates and spincoated at $3000 \mathrm{rpm}$ for 30 seconds. The samples were immediately placed inside a closed vessel with an atmosphere saturated in chloroform vapours for three hours to undergo solvent annealing. After this time, the samples were removed from the vessel and dried with a gentle nitrogen stream.

\subsection{Characterization}

The samples were imaged as prepared by field-emission scanning electron microscopy (SEM) in a Zeiss Merlin microscope with a Gemini II column, with operating electron energy of $1.2 \mathrm{keV}$. The block copolymer patterns were analysed by the directed self-assembly image analysis software developed in the laboratory applied to the collected SEM images. The code is written in Python ${ }^{(\mathrm{TM})}$ language for version 2.7.5, and was run in a conventional desktop terminal with Windows XP (Intel ${ }^{\circledR}$ Core $^{(\mathrm{TM})} 2$ CPU @ 1.86GHz and 3072MB RAM). Using this computational power, the time of analysis takes about 5 minutes for SEM image (1260x1260 pixel resolution). 


\section{Results and Discussion}

The block copolymer solution was spincoated over the silicon substrates and immediately solvent annealed (Figure 1a). As observable in Figure 1, this block copolymer shows spontaneous microphase segregation upon spincoating, resulting in cylinders perpendicular to substrate embedded in the PS matrix, resulting in the observable hexagonal dot array. PS-b-PEO systems are well-known for their good microphase segregation in brushless substrates. ${ }^{14}$

From the SEM image A in Figure 1b, it was possible to measure the critical dimensions of the PEO cylinders: diameter around $20 \mathrm{~nm}$ with a periodicity of approximately $40 \mathrm{~nm}$, consistent with reported previous works. ${ }^{5}$

After annealing in a saturated atmosphere of chloroform, the PEO cylinders started flipped the orientation towards parallel to substrate, resulting in line patterns. The cyclical flipping behaviour has been reported in this BCP system, but with toluene as annealing solvent. ${ }^{15}$ At the first hour of solvent annealing (Figure 1b.B), a few lines start to appear, indicating the flipping of the PEO cylinders. At three hours of solvent annealing, all the cylinders have flipped, thus only a compact array of lines is observed in SEM image B in Figure 1b. After 15 hours of solvent annealing time, the line array is conserved but a higher disorder is observed.

a)

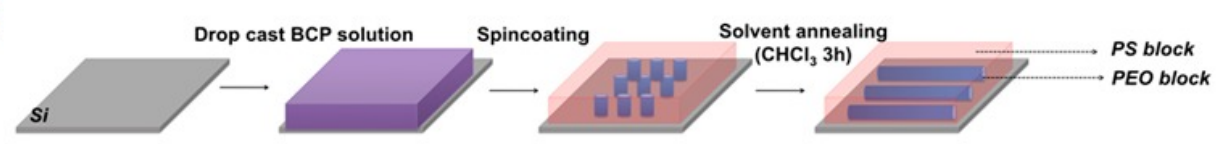

b)

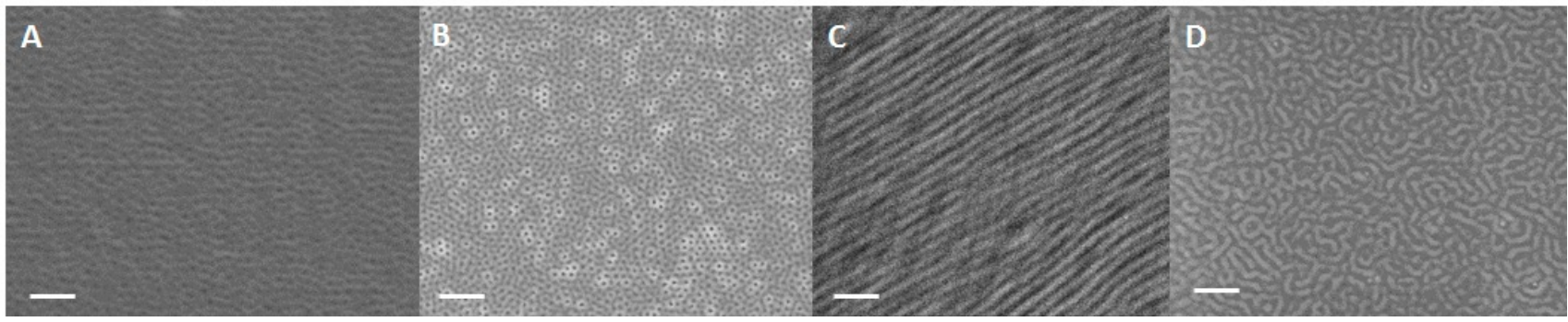

Figure 1. Schematic workflow of the preparation of line patterns from PS-b-PEO DSA (a) and FE-SEM images of the block copolymer samples as spun (A) and after solvent annealing 1 hour (B), 3 hours (C) and 15 hours (D)

In an effort to optimise the annealing time recipe in a systematic way, the line patterns recorded by FE-SEM were evaluated with our directed self-assembly image analysis software. The code has been developed to identify lines and defects specific from block copolymer self-assembly. In the first step, the region of interest is chosen in the SEM image. The first computational step identifies the elements (lines) and four type of DSA defects (lone or break points, branching, dislocation and turning points) and are colour-coded as shown in (Figure 2). 

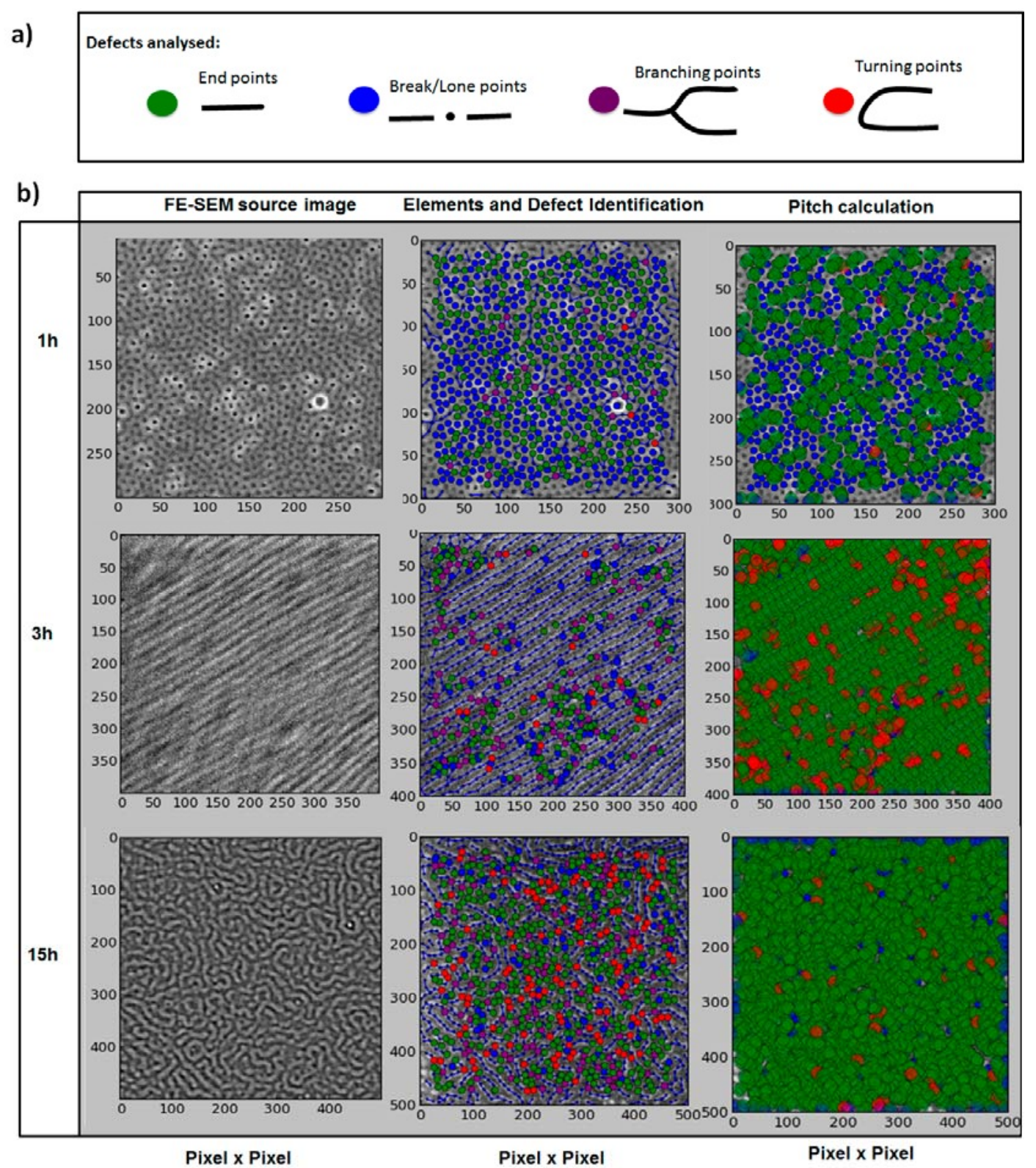

Figure 2. Left panel: original SEM images of the block copolymer samples after solvent annealing. Middle panel: software output of element (dark blue dashed dots) and defect identification (colour-coding: end points (green), lone points (blue), branch points (purple) and turn points (red)). Right panel: software output of pitch estimation graphic representation: the green and red dots represent the points used and excluded from the calculation, respectively.

The defects are identified as coloured dots and overlaid with the original SEM image and the marked lines, as seen in Figure 2. It should be noted that the dot arrays of PEO cylinders perpendicular to substrate are also identified as lone points. 
The collected statistics concerning the identification and quantification of the defects is summarized in Figure 3.

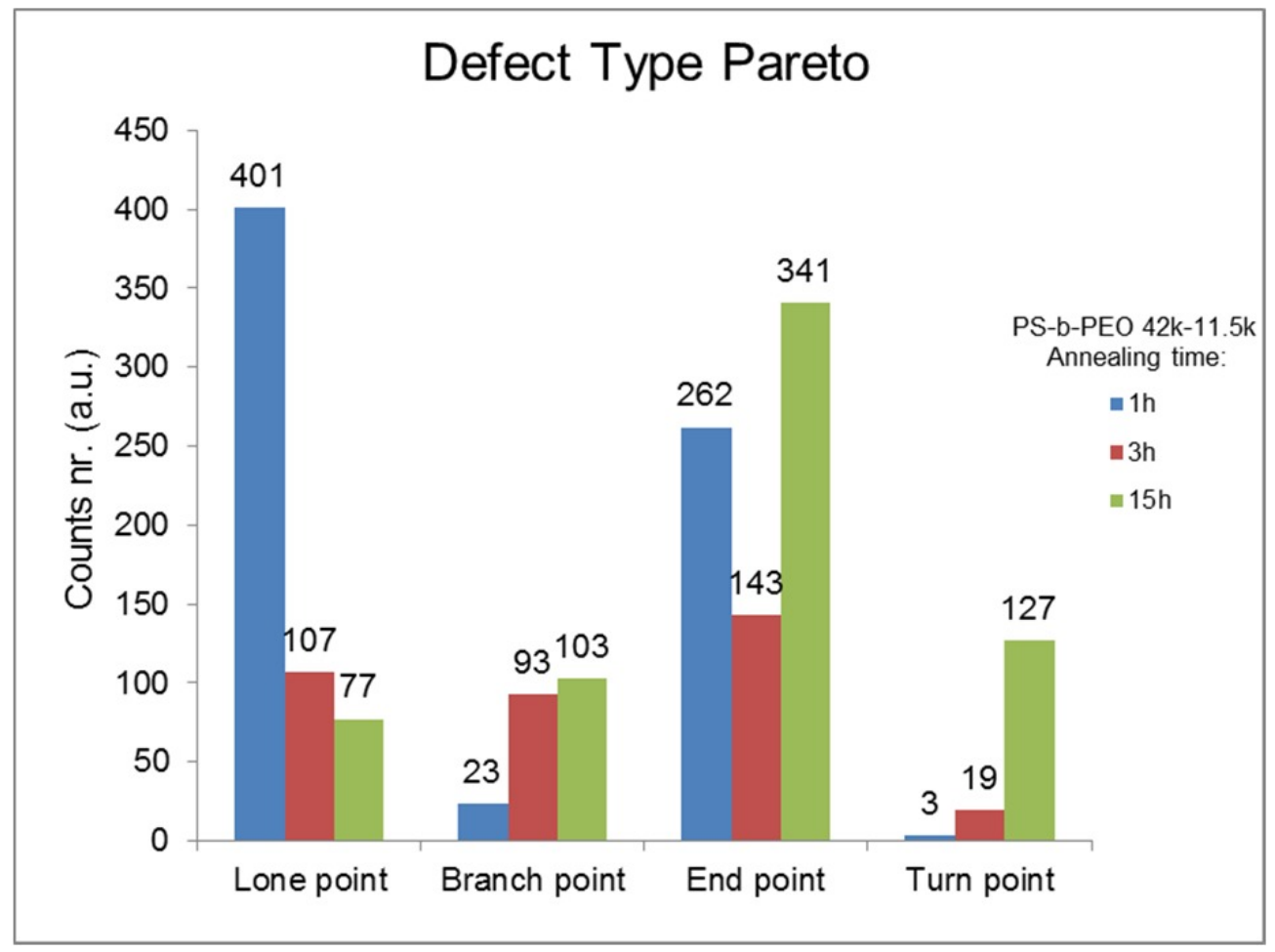

Figure 3. Type and number of defects found in the three samples.

With basis on the quantification of the four fundamental defects, the defect density can be defined as the total number of defects found in the analysed area, and was calculated for the three samples following Equation 1.

$$
\text { DefectDensity }=\frac{N r . \text { Branching }+N r . \text { Turning }+N r . \text { Lone }+N r E n d}{\text { Area }} \quad \text { Eq. } 1
$$

The image software extracts the pitch size distribution (Figure 3), and the estimated values and deviations collected are summarized in Table 2. The present methodology has been specially developed to calculate the pitch size, while the linewidths are indicative estimations. 
$1 \mathrm{~h}$

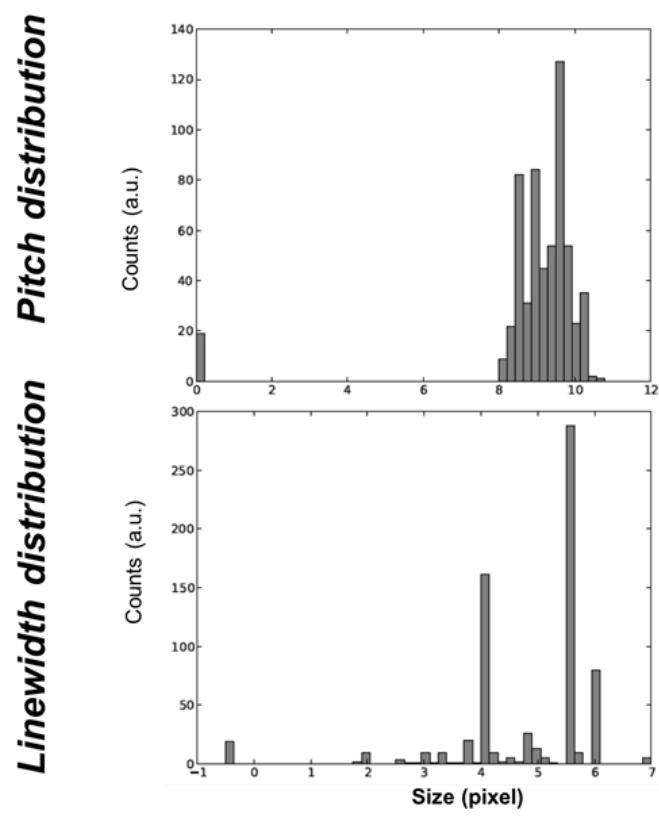

$3 h$
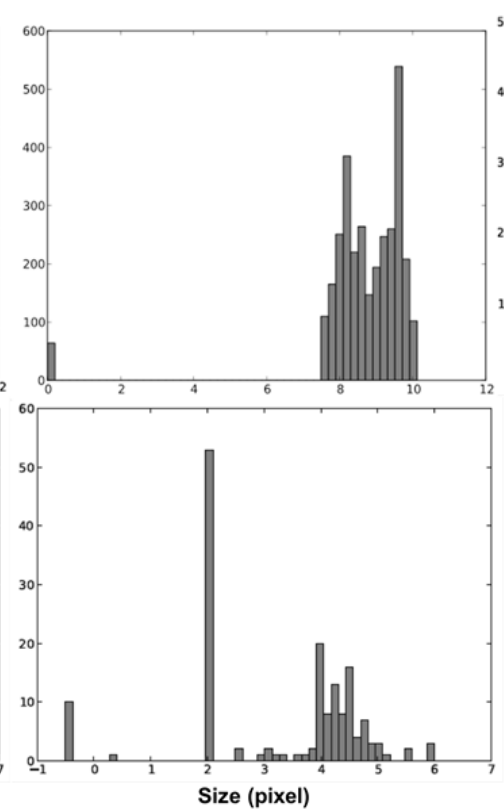

$15 \mathrm{~h}$

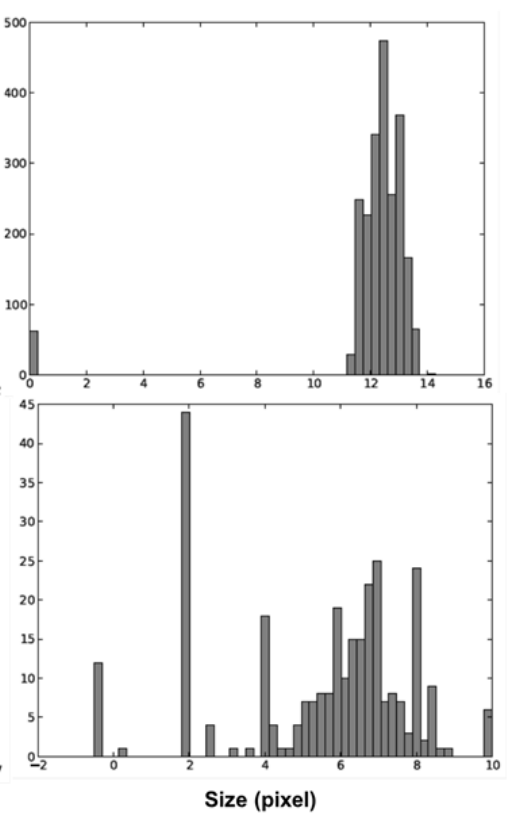

Figure 3. Pitch and linewidth distribution histograms calculated from the image analysis code for the three samples.

Table 2. Dimensional analysis of the line patterns by the image analysis methodology.

\begin{tabular}{|ccccc|}
\hline Annealing time & Pitch /nm & Pitch Placement error /nm & $\begin{array}{c}\text { Linewidth } \\
\text { estimation/nm }\end{array}$ & $\begin{array}{c}\text { Line lengths } \\
\text { /nm }\end{array}$ \\
\hline 1h & 39.4 & 4.7 & $19.6 \pm 5.4$ & 54.6 \\
3h & 43.5 & 4.2 & $20.1 \pm 3.2$ & 345.8 \\
15h & 39.4 & 6.6 & $18.2 \pm 7.8$ & 254.2 \\
\hline
\end{tabular}

The information gathered in the present methodology enables us to estimate the order of the linear pattern. If we consider the perfect order an array of fully straight lines, we can describe it as a100\% aligned line array. Thus, the alignment percentage of a linear array can be described as the density of turning points in relation to the total number of lines found, as defined in Equation 2. 


$$
\% \text { Alignment }=\left(1-\frac{N r . \text { Turns }}{\text { NrLines }}\right) \times 100 \quad \text { Eq. } 2
$$

The data concerning the defect analysis of the linear arrays investigated here are summarised in Table 3.

Table 3. Summary of number of elements, defects and estimated defect density in each sample.

\begin{tabular}{|cccccc|}
\hline \multicolumn{7}{|c|}{ DSA Line Pattern Defect Analysis } \\
Substrate & $\begin{array}{c}\text { Analysed } \\
\text { area }\left(\mathrm{cm}^{2}\right)\end{array}$ & $\begin{array}{c}\text { Total nr } \\
\text { lines }\end{array}$ & $\begin{array}{c}\text { Total nr } \\
\text { defects }\end{array}$ & $\begin{array}{c}\text { Defect Density } \\
\left(\mathrm{nr} \text { defects } \times 10^{9} / \mathrm{cm}^{2}\right)\end{array}$ & Alignment \% \\
\hline 1h & $2.53 \mathrm{E}-09$ & 24 & 689 & 272 & 88 \\
3h & $2.53 \mathrm{E}-09$ & 170 & 362 & 143 & 94 \\
\hline
\end{tabular}

In Figure 5, the defect density and the alignment percentage are graphically represented for a convenient comparison, with units as described in Table 2. It is observable that the lowest defect density and best aligned array corresponds to the PS-bPEO sample annealed for three hours under a saturated atmosphere of chloroform vapours. 


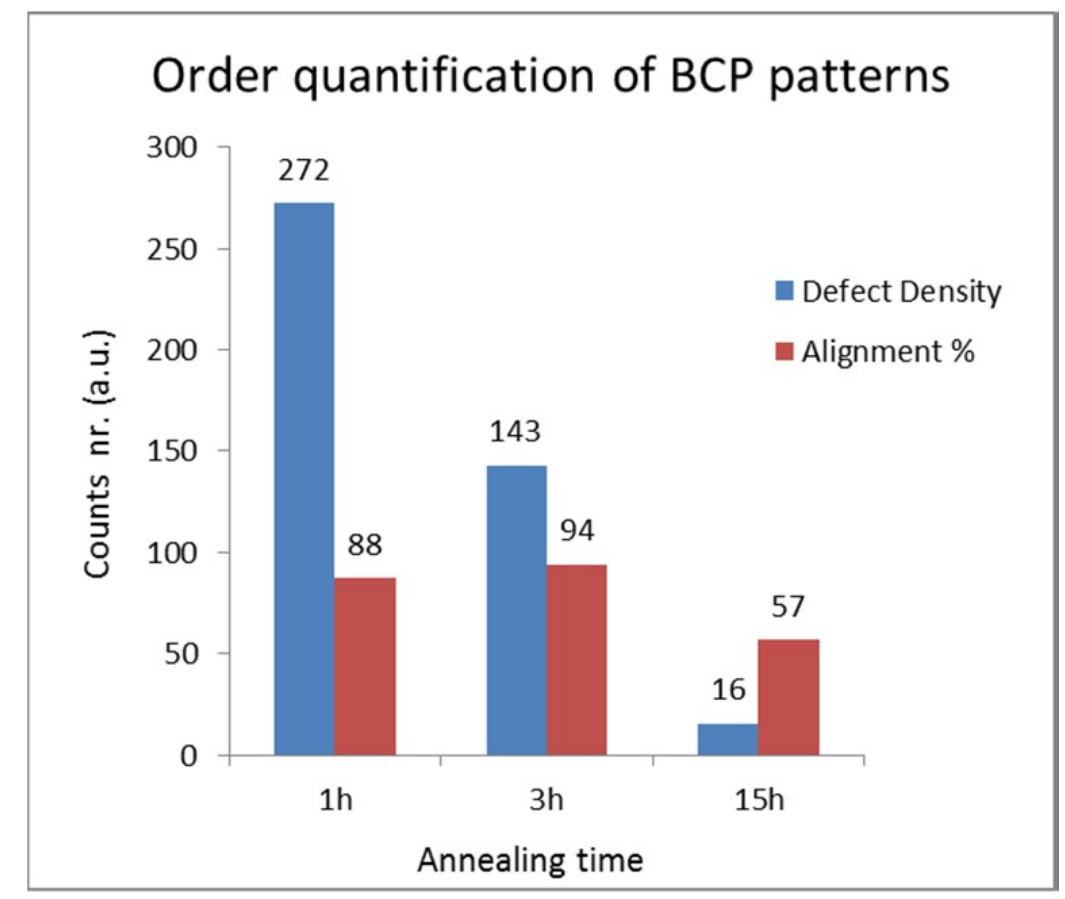

Figure 5. Comparison between defect density (nr defects $\mathrm{x} 10^{9} / \mathrm{cm}^{2}$ ) and alignment yield in percentage of the three samples studied.

\section{Conclusions}

The directed self-assembly of the hexagonal phase BCP PS-b-PEO 42k-11.5k treated with chloroform vapour results in PEO cylinders orientated parallel to substrate in a PS matrix. That orientation occurs along time and at three hours of annealing produces rather homogeneous line arrays. The line array examination was performed with the computational image analysis method that identifies the lines in the array along four types of defects. The dimensional analysis of the patterns yielded an approximation of the pitch size of the PS-b-PEO system used, giving an average $42 \mathrm{~nm}$ pitch size value with a standard deviation below $10 \%$. This calculation is rather good, given the simplicity of the method, the low computational power needed and the quality of the SEM images.

The defect analysis obtained from the method permitted estimating the defect density, making it possible to statistically quantify the order of the line patterns within the experimental error, the percentage of alignment, based on the density of turn points in the line array. The defect density and the percentage of alignment of the investigated samples demonstrate that the sample with higher order is the PS-b-PEO at 3 hours of solvent annealing, with defect density with $143 \times 10^{9}$ defects per $\mathrm{cm}^{2}$ with $94 \%$ alignment yield.

It has been demonstrated here that this technique is simple, versatile and powerful metrological tool for quality control of line arrays prepared by BCP DSA. Its fair accuracy combined with its simplicity and features dedicated to BCP DSA makes is it a valuable tool to standardize BCP DSA systems, which may be key for the successful implementation of the BCP lithography technology in the established semiconductor industry. 


\section{Acknowledgments}

The research leading to these results received funding from the European Union FP7 under the project LAMAND (grant agreement $n^{\circ}$ 245565), MERGING (grant agreement no. 309150) and QUANTIHEAT (grant agreement no. 604668), and the Spanish MINECO under the project TAPHOR (contract nr. MAT2012-31392) and the Science Foundation Ireland under grant number 09/SIRG/I1615. The contents of this work are the sole responsibility of the authors.

\section{References}

[1] Gronheid, R.; Van Look, L.; Rincon Delgadillo, P.; Ivan, P.; Cao, Y.; Lin, G.; Nealey, P. F., "High throughput grating qualification for rating directed self-assembly pattern performance using optical metrology" J. Photopolym. Sci. Technol. 26 (2), 147-152 (2013).

[2] Isawa, M.; Sakai, K.; Rincon Delgadillo, P. A.; Gronheid, R.; Yoshida, H. In Line edge roughness measurement technique for fingerprint pattern in block copolymer thin film, Proceedings of SPIE - The International Society for Optical Engineering, 2013; p 868114

[3] Rincon Delgadillo, P.; Harukawa, R.; Suri, M.; Durant, S.; Cross, A.; Nagaswami, V. R.; Van Den Heuvel, D.; Gronheid, R.; Nealey, P. In Defect source analysis of directed self-assembly process (DSA of DSA), Proceedings of SPIE - The International Society for Optical Engineering 8680, 2013; p 86800L

[4] Park, C.; Yoon, J.; Thomas, E. L., "Enabling nanotechnology with self assembled block copolymer patterns" Polym. 44 (22), 6725-6760 (2003).

[5] Simão, C.; Khunsin, W.; Salaün, M.; Zelsmann, M.; Morris, M. A.; Sotomayor Torres, C. M., "Order quantification of hexagonal periodic arrays fabricated by in situ solvent-assisted nanoimprint lithography of block copolymers" Nanotechnol. accepted, arXiv:1403.2250 (2014).

[6] Jung, Y. S.; Chang, J. B.; Verploegen, E.; Berggren, K. K.; Ross, C. A., "A path to ultranarrow patterns using self-assembled lithography" Nano Lett. 10 (3), 1000-1005 (2010).

[7] Gotrik, K. W.; Hannon, A. F.; Son, J. G.; Keller, B.; Alexander-Katz, A.; Ross, C. A., "Morphology control in block copolymer films using mixed solvent vapors" ACS Nano 6 (9), 8052-8059 (2012).

[8] Ghoshal, T.; Senthamaraikannan, R.; Shaw, M. T.; Holmes, J. D.; Morris, M. A., "In situ hard mask materials: A new methodology for creation of vertical silicon nanopillar and nanowire arrays" Nanoscale 4 (24), 7743-7750 (2012).

[9] Salaun, M.; Zelsmann, M.; Archambault, S.; Borah, D.; Kehagias, N.; Simao, C.; Lorret, O.; Shaw, M. T.; Torres, C. M. S.; Morris, M. A., "Fabrication of highly ordered sub-20 nm silicon nanopillars by block copolymer lithography combined with resist design" J. Mater. Chem. C 1 (22), 3544-3550.

[10] Borah, D.; Simao, C. D.; Senthamaraikannan, R.; Rasappa, S.; Francone, A.; Lorret, O.; Salaun, M.; Kosmala, B.; Kehagias, N.; Zelsmann, M.; Sotomayor-Torres, C. M.; Morris, M. A., "Soft-graphoepitaxy using nanoimprinted polyhedral oligomeric silsesquioxane substrates for the directed self-assembly of PS-b-PDMS" European Polymer Journal.

[11] Ouk Kim, S.; Solak, H. H.; Stoykovich, M. P.; Ferrier, N. J.; de Pablo, J. J.; Nealey, P. F., "Epitaxial selfassembly of block copolymers on lithographically defined nanopatterned substrates" Nature 424 (6947), 411-414 (2003).

[12] Simão, C.; Francone, A.; Borah, D.; Lorret, O.; Salaun, M.; Kosmala, B.; Shaw, M. T.; Dittert, B.; Kehagias, N.; Zelsmann, M.; Morris, M. A.; Sotomayor-Torres, C. M., "Soft graphoepitaxy of hexagonal PS-b- 
PDMS on nanopatterned POSS surfaces fabricated by nanoimprint lithography" J. Photopolym. Sci. Technol. 25 (2), 239-244 (2012).

[13] Sotomayor-Torres, C. M.; Simao, C.; Amann, A.; Tuchapsky, D.; Khunsin, W.; Morris, M. A. European Patent Application 2013.

[14] Mokarian-Tabari, P.; Collins, T. W.; Holmes, J. D.; Morris, M. A., "Brushless and controlled microphase separation of lamellar polystyrene-b-polyethylene oxide thin films for block copolymer nanolithography" J. Polym. Sci., Part B: Polym. Phys. 50 (13), 904-909 (2012).

[15] Mokarian-Tabari, P.; Collins, T. W.; Holmes, J. D.; Morris, M. A., "Cyclical "flipping" of morphology in block copolymer thin films" ACS Nano 5 (6), 4617-4623 (2011).

*claudia.simao@icn.cat; phone+34937371616; www.icn.cat/ p2n 